\title{
Associação da Síndrome do Ovário Policístico e neoplasias
}

\author{
Pedro Vinícius Duarte Fernandes ${ }^{1}$, Shara Sindel Gomes Silva ${ }^{2} \&$ Symara \\ Abrantes A. de Oliveira Cabral $^{2}$
}

(1) Universidade Federal de Campina Grande, Centro de Formação de Professores, Unidade Acadêmica de Ciências da Vida, Rua Sergio Moreira de Figueiredo, Cajazeiras, Paraíba, Brasil. Email: pedro.v.d.f@outlook.com

(2) Universidade Federal de Campina Grande, Centro de Formação de Professores, Unidade Acadêmica de Enfermagem, Rua Sergio Moreira de Figueiredo, Cajazeiras, Paraíba, Brasil. E-mail: sharasiindel@hotmail.com, symara_abrantes@hotmail.com

Fernandes P.V.D., Silva S.S.G. \& Cabral S.A.A.O. (2021) Associação da Síndrome do Ovário Policístico e neoplasias. Pesquisa e Ensino em Ciências Exatas e da Natureza, 5(edição especial 1): e1660. http://dx.doi.org/10.29215/pecen.v5i1.1660

Responsabilidade editorial: Maria Carmem Batista de Alencar. Recebido: 02 dezembro 2020. Aceito: 08 fevereiro 2021. Publicado: 26 fevereiro 2021.

Resumo: A Síndrome do Ovário Policístico (SOP) é um distúrbio endócrino que acomete as mulheres, apresentando-se como fator de risco pouco estudado, mas tendo complexa relação com os diversos subtipos de câncer, especialmente os do sistema reprodutor feminino, como o câncer de endométrio, ovário e mamas. No presente estudo, objetivou-se identificar a associação da SOP com neoplasias ginecológicas, através de uma revisão integrativa da literatura de caráter qualitativo. Os resultados indicam que, apesar de haver conexões entre a síndrome e o desenvolvimento de câncer ginecológico, é necessário maior investigação para uma afirmação conclusiva de tal associação. Os resultados também ressaltam a importância do tratamento precoce da SOP para que haja redução de fatores de risco para neoplasias.

Palavras-chave: Ovários policísticos, distúrbio hormonal, neoplasias ginecológicas, câncer.

\section{Association of Polycystic Ovary Syndrome and neoplasms}

Abstract: Polycystic Ovary Syndrome (PCOS) presents itself as an endocrine disorder that affects women, presenting itself as a poorly studied risk factor, but with a complex relationship with the different subtypes of cancer, especially those of the female reproductive system, such as endometrial, ovarian and breast cancer. The present study aimed to identify the association of PCOS with gynecological neoplasms, through an integrative review of the qualitative literature. The results indicate that, although there are connections between the syndrome and the development of gynecological cancer, further investigation is necessary for a conclusive statement of such an association, it also highlights the importance of early treatment of PCOS in order to reduce risk factors for neoplasms.

Keywords: Polycystic ovaries, hormonal disorder, gynecological neoplasms, cancer.

A Síndrome do Ovário Policístico (SOP) configura-se como uma disfunção endócrina de causa multifatorial que acomete as mulheres, principalmente na idade reprodutiva. A SOP atinge cerca de 5 a $10 \%$ das mulheres em idade fértil, podendo ser caracterizada por hiperandrogenismo, complicações metabólicas e reprodutivas, o que pode ocasionar multimorbidades, como diabetes tipo 2, obesidade, hipertensão arterial sistêmica, problemas cardiovasculares, acne, além de que, em casos mais graves, caso não seja tratado corretamente, poderá evoluir para um câncer ginecológico (Faria et al. 2013). 
Para o diagnóstico da SOP faz-se necessário o estabelecimento de, pelo menos, duas ou mais manifestações da síndrome, dentre elas: oligovulação ou anovulação crônica, aspectos de hiperandrogenismo, como acne e excesso de pelos, e a presença de cistos ovarianos detectados na ultrassonografia (Andrade et al. 2016).

Importante se faz comiserar que a SOP é uma condição que repercute de forma negativa na vida da mulher, afetando diversos campos, saúde física e mental, porém o aumento da possiblidade de neoplasias é algo que deve ser investigado e identificado. É nesse contexto que se faz necessário uma pesquisa aprofundada acerca do tema para contribuir positivamente para a prevenção de neoplasias nesse grupo. Desse modo, o presente artigo busca identificar a associação da SOP e o desencadeamento de câncer ginecológico na literatura.

Trata-se de uma revisão de literatura integrativa, de caráter qualitativo, a qual o levantamento bibliográfico foi realizado no mês de outubro de 2020, utilizando as bases de dados eletrônicas: SciELO, Biblioteca Virtual da Saúde (BVS), Medline, Lilacs e PubMed. Os seguintes descritores foram utilizados: "Polycystic ovary syndrome AND neoplasms". Como critérios de inclusão considerou-se: artigos completos nos idiomas português, inglês e espanhol; e exclusão: artigos não atendendo ao objetivo do trabalho, trabalhos de conclusão de curso e resumos.

Utilizando as estratégias e ferramentas de busca foram identificados um total de 932 artigos, sendo que 791 não atendiam a temática em pauta, de tal modo que 141 trabalhos tiveram seus títulos e resumos lidos, sendo selecionados apenas 16 artigos, conforme Figura 1. Os artigos selecionados foram lidos na íntegra, conforme Quadro 1.

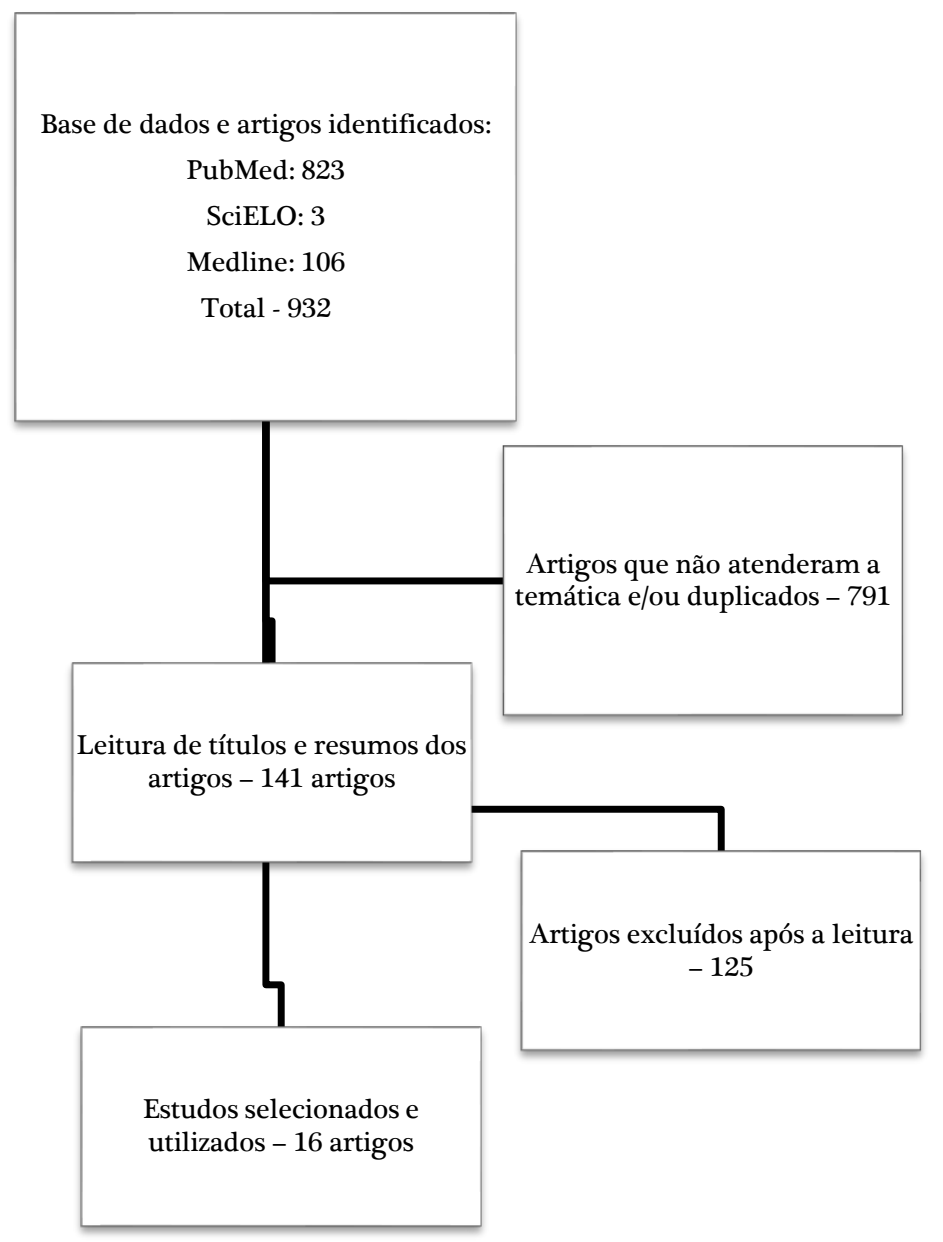

Figura 1. Fluxograma de seleção dos estudos. 


\section{Ovário Policístico e neoplasias}

Quadro 1. Análise conceitual dos estudos analisados nesta revisão.

\begin{tabular}{|c|c|c|c|}
\hline Autor(es), ano & Título & Objetivo & Resultados \\
\hline $\begin{array}{l}\text { Andrade et al. } \\
\quad(2016)\end{array}$ & $\begin{array}{l}\text { Aspectos atuais da SOP: uma } \\
\text { revisão da literatura. }\end{array}$ & $\begin{array}{l}\text { O objetivo deste artigo foi apresentar uma } \\
\text { revisão da literatura relacionada a SOP, } \\
\text { incluindo sua patogênese, manifestações } \\
\text { clínicas, diagnóstico e aspectos terapêuticos, } \\
\text { bem como sua associação com } \\
\text { multimorbidades. }\end{array}$ & $\begin{array}{l}\text { Mulheres com SOP, além de anovulação e } \\
\text { infertilidade, apresentam risco aumentado de } \\
\text { desenvolver hipertensão e doença cardiovascular } \\
\text { em associação com síndrome metabólica. }\end{array}$ \\
\hline Cirillo et al. (2016) & $\begin{array}{l}\text { Menstruação irregular prediz } \\
\text { câncer de ovário: evidências } \\
\text { prospectivas dos Estudos de } \\
\text { Saúde e Desenvolvimento } \\
\text { Infantil. }\end{array}$ & $\begin{array}{l}\text { Identificar se a menstruação irregular prediz } \\
\text { menor risco de câncer de ovário, devido à } \\
\text { ovulação menos frequente. }\end{array}$ & $\begin{array}{l}\text { A associação com ciclos irregulares foi substancial } \\
\text { e significativa para morte serosa de alto grau } \\
\text { considerável, mas marginalmente significativa } \\
\text { para incidência serosa de alto grau. }\end{array}$ \\
\hline $\begin{array}{l}\text { Xiangyan et al. } \\
\text { (2018) }\end{array}$ & $\begin{array}{l}\text { Por que a SOP precisa de } \\
\text { tratamento de longo prazo? }\end{array}$ & $\begin{array}{l}\text { A importância de um tratamento de longo } \\
\text { prazo para a SOP. }\end{array}$ & $\begin{array}{l}\text { Há um consenso de que o risco de câncer } \\
\text { endometrial pode ser aumentado. A resistência à } \\
\text { insulina, importante na fisiopatologia da SOP, } \\
\text { predispõe os pacientes à disfunção metabólica e } \\
\text { aumento do risco de diabetes mellitus tipo } 2 \text {. }\end{array}$ \\
\hline $\begin{array}{l}\text { Hobeiri \& Jenabi } \\
\quad(2016)\end{array}$ & $\begin{array}{l}\text { A associação entre a SOP e } \\
\text { câncer de mama: uma meta- } \\
\text { análise. }\end{array}$ & $\begin{array}{l}\text { Estimar a associação entre a SOP e o risco de } \\
\text { câncer de mama. }\end{array}$ & $\begin{array}{l}\text { A associação entre SOP e o risco de câncer de } \\
\text { mama em estudos de caso-controle foi estimada } \\
\text { em } 0.87 \text { e em estudos de coorte foi estimada em } \\
1.18 \text {. }\end{array}$ \\
\hline Faria et al. (2013) & $\begin{array}{l}\text { SOP e fatores relacionados em } \\
\text { adolescentes de } 15 \text { a } 18 \text { anos. }\end{array}$ & $\begin{array}{l}\text { Avaliar os fatores relacionados à presença da } \\
\text { SOP em adolescentes. }\end{array}$ & $\begin{array}{l}\text { Foram avaliados } 485 \text { adolescentes, com idade } \\
\text { média de } 16.3 \pm 0.9 \text { anos. A prevalência de SOP foi } \\
\text { de } 6.2 \% \text {. Não se encontrou diferença entre os } \\
\text { grupos quanto aos parâmetros antropométricos e } \\
\text { tempo de uso de anticoncepcionais, porém houve } \\
\text { diferença quanto à idade da menarca }(\mathrm{p}<0.004) \text {, e } \\
\text { a mais tardia foi fator de proteção para síndrome. }\end{array}$ \\
\hline Harris et al. (2017) & $\begin{array}{l}\text { Ciclos menstruais longos e } \\
\text { irregulares, síndrome dos } \\
\text { ovários policísticos e risco de } \\
\text { câncer de ovário em um estudo } \\
\text { caso-controle de base } \\
\text { populacional. }\end{array}$ & $\begin{array}{l}\text { Identificar características do ciclo menstrual e } \\
\text { SOP autorreferidas associadas ao risco de } \\
\text { câncer de ovário entre } 2.041 \text { mulheres com } \\
\text { câncer de ovário epitelial. }\end{array}$ & $\begin{array}{l}\text { A irregularidade do ciclo menstrual foi associada a } \\
\text { uma diminuição do risco de tumores serosos de } \\
\text { alto grau, mas a um risco aumentado de tumores } \\
\text { serosos limítrofes entre mulheres que nunca } \\
\text { usaram COs e aquelas que estavam acima do peso. }\end{array}$ \\
\hline $\begin{array}{l}\text { Spritzer et al. } \\
\quad(2005)\end{array}$ & \begin{tabular}{llr} 
Neoplasias & \multicolumn{2}{c}{ associadas $\quad$ à } \\
síndrome & dos $\quad$ ovários \\
policísticos. & &
\end{tabular} & $\begin{array}{l}\text { Investigar se a longo prazo, pacientes com SOP } \\
\text { possam apresentar maior risco de neoplasias do } \\
\text { trato reprodutivo como carcinoma (CA) de } \\
\text { endométrio, mama e ovário. }\end{array}$ & $\begin{array}{l}\text { O ciclo irregular na SOP pode ser fator de risco } \\
\text { para neoplasias ginecológicas, porém se faz } \\
\text { necessário mais estudos para uma afirmação } \\
\text { conclusiva. }\end{array}$ \\
\hline $\begin{array}{l}\text { Valejo \& Tiezzi } \\
\quad(2009)\end{array}$ & $\begin{array}{l}\text { Tratamento do câncer de } \\
\text { endométrio. }\end{array}$ & $\begin{array}{l}\text { Objetivou-se discutir algumas evidências em } \\
\text { relação a cada tipo de tratamento. }\end{array}$ & $\begin{array}{l}\text { Com base nas evidências, o tratamento do } \\
\text { carcinoma de endométrio deve ser conduzido de } \\
\text { acordo com o estadiamento e a presença de fatores } \\
\text { de risco. }\end{array}$ \\
\hline Yin et al. (2019) & $\begin{array}{l}\text { Associação entre a SOP e o risco } \\
\text { de câncer. }\end{array}$ & $\begin{array}{l}\text { Investigar a associação da SOP e o risco para } \\
\text { câncer com uma população de mulheres com } \\
\text { idades entre } 15 \text { e } 50 \text { anos entre } 1985 \text { e } 2009 \\
\end{array}$ & $\begin{array}{l}\text { Nenhuma interação significativa entre SOP e } \\
\text { covariáveis foi observada. }\end{array}$ \\
\hline Pollak (2012) & $\begin{array}{l}\text { Investigando metformina para } \\
\text { prevenção e tratamento do } \\
\text { câncer: o fim do começo. }\end{array}$ & $\begin{array}{l}\text { Objetivou-se investigar se a exposição à } \\
\text { metformina está associada com risco reduzido } \\
\text { de câncer e/ou melhor prognóstico }\end{array}$ & $\begin{array}{l}\text { Identificou-se que o uso da metformina ajuda na } \\
\text { prevenção e tratamento devido ao controle do } \\
\text { perfil glicêmico. }\end{array}$ \\
\hline $\begin{array}{c}\text { Ignatov \& } \\
\text { Ortmann }(2020)\end{array}$ & $\begin{array}{l}\text { Fatores de risco endócrino de } \\
\text { câncer de endométrio: Síndrome } \\
\text { dos ovários policísticos, } \\
\text { anticoncepcionais } \quad \text { orais, } \\
\text { infertilidade, tamoxifeno. }\end{array}$ & $\begin{array}{l}\text { O objetivo desta revisão foi investigar a relação } \\
\text { entre infertilidade, síndrome dos ovários } \\
\text { policísticos (SOP), OCs e tamoxifeno (TAM) e o } \\
\text { risco de câncer endometrial. }\end{array}$ & $\begin{array}{l}\text { A maioria dos dados publicados sobre a associação } \\
\text { entre infertilidade e SOP são inconclusivos, } \\
\text { enquanto o efeito do tamoxifeno no risco de câncer } \\
\text { endometrial foi bem estabelecido. }\end{array}$ \\
\hline $\begin{array}{l}\text { Shafiee et al. } \\
\quad(2017)\end{array}$ & $\begin{array}{l}\text { A expressão do gene da proteína } \\
-1 \text { de ligação do elemento } \\
\text { regulador de esterol (SREBP1) é } \\
\text { igualmente aumentada na SOP e } \\
\text { câncer endometrial. }\end{array}$ & $\begin{array}{l}\text { O objetivo deste estudo foi investigar a } \\
\text { expressão do gene da proteína-1 de ligação do } \\
\text { elemento regulador do esterol endometrial na } \\
\text { SOP e câncer endometrial do endométrio, e } \\
\text { correlacionar a expressão do gene da proteína- } \\
1 \text { de ligação do elemento regulador do esterol } \\
\text { endometrial com perfis de lipídios séricos. }\end{array}$ & $\begin{array}{l}\text { A expressão do gene da proteína-1 de ligação do } \\
\text { elemento regulador de esterol é significativamente } \\
\text { aumentada no endométrio de mulheres com SOP e } \\
\text { mulheres com câncer endometrial. }\end{array}$ \\
\hline Moran et al. (2015) & 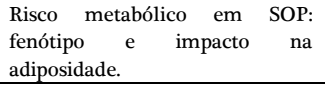 & Identificar risco metabólico na SOP. & $\begin{array}{l}\text { A SOP está associada à piora da saúde } \\
\text { cardiometabólica. }\end{array}$ \\
\hline Jiao et al. (2019) & $\begin{array}{l}\text { As características genéticas e } \\
\text { epigenéticas em tecidos } \\
\text { ovarianos de pacientes com SOP } \\
\text { com menstruação irregular } \\
\text { assemelham-se às do câncer de } \\
\text { ovário }\end{array}$ & $\begin{array}{l}\text { Investigar se há características genéticas e } \\
\text { epigenéticas entre SOP com menstruação } \\
\text { irregular e câncer de ovário. }\end{array}$ & $\begin{array}{l}\text { Foi identificado múltiplas mutações pontuais em } \\
\text { ambos os genes BRCA1 e MLH1 em pacientes com } \\
\text { menstruação irregular e previmos a } \\
\text { patogenicidade potencial dessas mutações, ou seja, } \\
\text { a menstruação irregular proporciona risco } \\
\text { aumentado de câncer de ovário. }\end{array}$ \\
\hline Harris et al. (2018) & $\begin{array}{l}\text { SOP, Oligomenorréia e } \\
\text { Histótipos de Risco de Câncer de } \\
\text { Ovário: Evidências do câncer de } \\
\text { ovário. }\end{array}$ & $\begin{array}{l}\text { Objetivou-se estudar uma amostra de } 16.594 \\
\text { mulheres com câncer de ovário invasivo }(\mathrm{n}= \\
\text { 13.719) ou doença ovariana limítrofe }(\mathrm{n}= \\
2.875) \text { e } 17.718 \text { controles em busca de } \\
\text { evidências de câncer de ovário. }\end{array}$ & $\begin{array}{l}\text { Características do ciclo menstrual influenciam o } \\
\text { risco de câncer ovariano de forma diferenciada } \\
\text { com base no histótipo. }\end{array}$ \\
\hline $\begin{array}{l}\text { Harris \& Terry } \\
\quad(2016)\end{array}$ & $\begin{array}{l}\text { SOP e risco de câncer de } \\
\text { endométrio, ovário e mama: } \\
\text { uma revisão sistemática. }\end{array}$ & $\begin{array}{l}\text { Objetivou-se investiga se o ambiente } \\
\text { metabólico e hormonal alterado entre } \\
\text { mulheres com SOP pode aumentar o risco de } \\
\text { alguns tipos de câncer. }\end{array}$ & $\begin{array}{l}\text { A associação com câncer de ovário foi menos clara, } \\
\text { mas um risco potencialmente aumentado do } \\
\text { subtipo seroso limítrofe foi relatado por dois } \\
\text { estudos. Nenhuma associação consistente entre o } \\
\text { risco de SOP e câncer de mama foi observada. }\end{array}$ \\
\hline
\end{tabular}


A SOP é uma síndrome metabólica que possui uma apresentação clínica já citada e está associada a sobrepeso, dislipidemias, Diabetes tipo II, resistência à insulina, sendo tal síndrome importante causa no que diz respeito a infertilidade. De acordo com Harris et al. (2016), fica claro que a associação entre SOP e outros tipos de câncer se faz necessária, uma vez que, identificada como fator de risco pode reduzir o número de morbidades associadas ao câncer. Por outro lado, enfrenta-se um entrave metodológico no que diz respeito ao manejo das variáveis que podem afetar o resultado de muitas pesquisas, sendo necessário um aprofundamento dos estudos dessa relação.

Segundo Valejo \& Tiezzi (2009) o câncer de endométrio é a neoplasia mais comum em países desenvolvidos, geralmente acomete as mulheres na pós-menopausa. Os fatores de risco para o desenvolvimento desta neoplasia são: obesidade, diabetes, uso de anticoncepcionais e terapia de estrogênio.

O câncer de ovário, por sua vez, constitui-se como a segunda neoplasia ginecológica mais frequente no Brasil, havendo diversos fatores que podem levar ao surgimento, como a idade avançada, infertilidade, obesidade e fatores genéticos. Já o câncer de mama é causado pela multiplicação desordenada das células da mama, este também pode ocorrer em homens, porém são raros os casos, com os fatores de riscos se repetindo, de maneira geral, aos cânceres de caráter ginecológico (INCA 2020).

Yin et al. (2019) em estudo de coorte com um grupo de 3.493 .604 mulheres, com idades entre 15 e 50 anos, no período de 1985 e 2009, evidenciaram que 14.764 mulheres apresentaram critérios diagnósticos de SOP, delas 1.2\% (182) apresentaram estágios primários de câncer, 48 câncer de mama e 40 câncer do aparelho reprodutivo feminino, incluindo ovário e endométrio. É interessante ressaltar que o estudo levou em consideração variáveis como a predisposição genética e a exposição ambiental que poderiam influir no resultado da pesquisa, configurando-se uma base para compreender a SOP como fator de risco para subtipos de câncer.

Algumas considerações acerca do mecanismo de ação que pode predispor ao câncer de ovário aos pacientes portadores de SOP é a alta exposição ao estrogênio durante longos períodos de anovulação, ou seja, o aumento do risco pode estar associado a alta estimulação da multiplicação celular pelo estrogênio. É nesse contexto que cabe citar a ação protetora dos contraceptivos orais, como estudado por Harris et al. (2016), e da metformina, como citado por Pollak (2012).

O câncer de endométrio também vale a pena ser abordado, uma vez que apresenta vários fatores de risco endócrinos, dentre eles é possível citar menarca precoce, menopausa tardia, nuliparidade, infertilidade e altos níveis de estrogênio. Esse último ponto é de fundamental importância, uma vez que a SOP induz longos períodos de anovulação com altos níveis séricos de estrogênio. Como fatores protetores apresenta-se mais uma vez o uso de contraceptivos orais e o aumento do número de filhos (Ignatov \& Ortmann 2020).

Ainda nessa abordagem faz-se fundamental discorrer acerca da relação entre marcadores genéticos que relacionam o câncer endometrial e a SOP, como estudado por Shafiee et al. (2017). O presente estudo demonstrou que o gene Sterol regulatory element binding protein-1 (SREBP1) tem sua expressão aumentada em mulheres acometidas por SOP e câncer endometrial quando comparada com mulheres sem as duas enfermidades. Uma possível hipótese está ligada ao fato de o gene ser responsável pela regulação da síntese lipídica, sendo pertinente observar que dislipidemias e síndrome metabólica fazem-se presentes como fatores de risco tanto na SOP quanto no câncer endometrial (Moran et al. 2015).

Outro ponto prioritário em relação as neoplasias malignas é o câncer de ovário, pois esse subtipo está fortemente associado a desfechos mórbidos nos pacientes acometidos por essa enfermidade. Uma explicação plausível seria o diagnóstico em um estado já avançado da doença devido ao quadro oligossintomático manifestado nos primeiros estágios da evolução desse câncer. É nesse contexto em que se nota a importância da determinação dos fatores de risco, visando uma abordagem preventiva do câncer. A SOP apresenta-se como um fator de risco ainda pouco estudado e que tem uma complexa relação com os diversos subtipos de câncer especialmente os do sistema reprodutor feminino. Assim, com base nos estudos realizados por 
Jiao et al. (2019), há uma relação genética e epigenética entre os tecidos de pacientes com SOP e com câncer de ovário, estando também os genes BRCA 1 e MLH1 mutados em múltiplos pontos em pacientes com o ciclo menstrual irregular, predispondo estes a outros tipos de câncer.

Em estudo realizado por Harris et al. (2017), evidencia-se que ciclos menstruais irregulares ou longos estão associados a menores chances de desenvolver carcinomas infiltrativos ovarianos, todavia estes critérios estão associados ao aumento do risco de tumores serosos ovarianos, como corroborado por Harris et al. (2019), fazendo-se necessário um aprofundamento no que diz respeito a coleta de dados e correção de fatores externo que podem influenciar os resultados.

O câncer de mama também é um importante tópico quando se abordam as neoplasias mais comuns em mulheres, na realidade é o tipo de câncer mais diagnosticado de todos os carcinomas. Entretanto a relação entre SOP e o Ca de mama ainda carece de estudos e uma investigação mais aprofundada (Spritzer et al. 2005).

Em estudo realizado por Hobeiri \& Jenabi (2016), buscou-se evidenciar uma possível relação entre SOP e câncer de mama. No presente estudo, utilizando-se dos métodos de metanálise e coorte, evidenciou-se que não há aumento dos casos de carcinoma de mama devido a SOP. Por outro lado, mostrou-se uma relação diretamente proporcional entre esse tipo de câncer e a obesidade.

Por fim, deve-se entender que o tratamento para SOP, atualmente, é acessível e baseado em contraceptivos orais, com efeito protetor massivo sobre os subtipos de cânceres ginecológicos. A importância do tratamento precoce da SOP diz respeito a redução de fatores de risco para neoplasias, contudo também se faz necessário um tratamento a longo prazo com um bom acompanhamento endocrinológico, uma vez que a obesidade, síndrome metabólica, resistência à insulina e diabetes mellitus tipo 2 podem surgir nesses pacientes. Nessa abordagem, uma dieta equilibrada, uso de metformina, atividades físicas regulares, uso de contraceptivos orais e o uso de orlistate, quando utilizados de acordo com as prioridades individuais de cada paciente, podem atuar como boas medidas no manejo da SOP e evitar neoplasias ginecológicas (Xiangyan et al. 2018).

\section{Conclusão}

Constata-se que as atuais evidências não fundamentam uma afirmação conclusiva no que se diz respeito a associação da SOP com neoplasias, devido ao pequeno número de casos, porém, é notório a associação do ciclo irregular para risco de câncer ginecológico. Portanto, é necessário que haja maior investigação sobre o assunto para maior esclarecimento da associação e maior atenção no tratamento e prevenção de possíveis agravos nas mulheres com a SOP.

\section{Referências}

Andrade V.H.L., Mata A.M.O.F., Borges R.S., Costa-Silva D.R., Martins L.M., Ferreira P.M.P., Cunha-Nunes L.C. \& Silva B.B. (2016) Aspectos atuais da síndrome do ovário policístico: uma revisão da literatura. Revista da Associação Médica Brasileira, 62(9): 867-871. https://doi.org/10.1590/1806-9282.62.09.867

Cirillo P.M., Wang E.T., Cedars M.I., Chen L-m \& Cohn B.A. (2016) Irregular menses predicts ovarian cancer: Prospective evidence from the Child Health and Development Studies. International Journal of Cancer, 139(5): 1009-1017. https://doi.org/10.1002/ijc.30144

Faria F.R., Gusmão L.S., Faria E.R., Gonçalves V.S.S., Cecon R.S., Franceschini S.C.C. \& Priore S.E. (2013) Síndrome do ovário policístico e fatores relacionados em adolescentes de 15 a 18 anos. Revista da Associação Médica Brasileira, 59(4): 341-346. https://doi.org/10.1016/j.ramb.2013.02.003

Harris H. \& Terry K. (2016) Polycystic ovary syndrome and risk of endometrial, ovarian, and breast cancer: a systematic review. Fertility Research and Practice, 2016 2:14. https://doi.org/10.1186/s40738-016-0029-2 
Harris H.R., Titus L.J., Cramer D.W. \& Terry K.L. (2017) Long and irregular menstrual cycles, polycystic ovary syndrome, and ovarian cancer risk in a population-based case-control study. International Journal of Cancer, 140: 285-291. https://doi.org/10.1002/ijc.30441

Harris H.R., Babic A., Webb P.M., Nagle C.M., Jordan S.J., Risch H.A., Rossing M.A., Doherty J.A., Goodman M.T., Modugno F., Ness R.B., Moysich K.B., Kjær S.K., Høgdall E., Jensen A., Schildkraut J.M., Berchuck A., Cramer D.W., Bandera E.V., Wentzensen N., Kotsopoulos J., Narod S.A., Phelan C.M., McLaughlin J.R., Anton-Culver H., Ziogas A., Pearce C.L., Wu A.H. \& Terry K.L. (2018) Polycystic Ovary Syndrome, Oligomenorrhea, and Risk of Ovarian Cancer Histotypes: Evidence from the Ovarian Cancer Association Consortium. Cancer Epidemiology, Biomarkers \& Prevention, 27(2): 174-182. https://doi.org/10.1158/1055-9965.EPI-17-0655

Harris H.R., Cushing-Haugen K.L., Webb P.M., Nagle C.M., Jordan S.J., Risch H.A., Rossing M.A., Doherty J.A., Goodman M.T., Modugno F., Ness R.B., Moysich K.B., Kjær S.K., Høgdall E., Jensen A., Schildkraut J.M., Berchuck A., Cramer D.W., Bandera E.V., Rodriguez L., Wentzensen N., Kotsopoulos J., Narod S.A., McLaughlin J.R., Anton-Culver H., Ziogas A., Pearce C.L., Wu A.H., Lindström S. \& Terry K.L. (2019) Association between genetically predicted polycystic ovary syndrome and ovarian cancer: a mendelian randomization study. International Jornal of Epidemiology, 48(3): 822-830. https://doi.org/10.1093/ije/dyz113

Ignatov A. \& Ortmann O. (2020) Endocrine Risk Factors of Endometrial Cancer: Polycystic Ovary Syndrome, Oral Contraceptives, Infertility, Tamoxifen. Cancers, 12(7): 1766. https://doi.org/10.3390/cancers12071766

INCA (2020) Instituto Nacional do Câncer. Estimativas 2000. Ministério da Saúde. Disponível em: http://www.inca.org.br/releases/press/estimativa-2000.html (Acesso em: 18/10/2020).

Jiao J., Sagnelli M., Shi B., Fang Y., Shen Z., Tang T., Dong B., Li D. \& Wang X. (2019) Genetic and epigenetic characteristics in ovarian tissues from polycystic ovary syndrome patients with irregular menstruation resemble those of ovarian cancer. BMC Endocrine Disorders, 19(30). https://doi.org/10.1186/s12902-019-0356-5

Moran L.J., Norman R.J. \& Teede H.J. (2015) Metabolic risk in PCOS: phenotype and adiposity impact. Trends in Endocrinology \& Metabolism, 26(3): 136-143.

https://doi.org/10.1016/j.tem.2014.12.003

Pollak M.N. (2012) Investigating metformin for cancer prevention and treatment: the end of the beginning. Cancer Discovery, 2(9): 778-790. https://doi.org/10.1158/2159-8290.CD-12-0263

Shafiee M.N., Mongan N., Seedhouse C., Chapman C., Deen S., Abu J. \& Atiomo W. (2017) Sterol regulatory element binding protein-1 (SREBP1) gene expression is similarly increased in polycystic ovary syndrome and endometrial cancer. Acta Obstetricia et Gynecologica Scandinavica, 96(5): 556-562. https://doi.org/10.1111/aogs.13106

Shobeiri F. \& Jenabi E. (2016) The association between polycystic ovary syndrome and breast cancer: a meta-analysis. Obstetrics \& Gynecology Science, 59(5): 367-372. https://doi.org/10.5468/ogs.2016.59.5.367

Spritzer P.M., Morsch D.M. \& Wiltgen D. (2005) Neoplasias associadas à síndrome dos ovários policísticos. Arquivos Brasileiros de Endocrinologia \& Metabologia, 49(5): 805-810. http://dx.doi.org/10.1590/S0004-27302005000500022

Spritzer P.M., Morsch D.M. \& Wiltgen D. (2020) Neoplasias associadas à síndrome dos ovários policísticos. Arquivos Brasileiros de Endocrinologia \& Metabologia, 49(5): 805-810. http://dx.doi.org/10.1590/S0004-27302005000500022

Valejo F.A.M. \& Tiezzi D.G. (2009) Tratamento do câncer de endométrio. Revista Feminina, 37(11): 603-610

Xiangyan R., Meng L. \& Alfred M.O. (2018) Por que a síndrome do ovário policístico (SOP) precisa de tratamento de longo prazo? Current Pharmaceutical Design, 24(39): 4685-4692. https://doi.org/10.2174/1381612825666190130104922

Yin W., Falconer H., Yin L., Xu L. \& Ye W. (2019) Association Between Polycystic Ovary Syndrome and Cancer Risk. JAMA Oncology, 5(1): 106-107.

https://doi.org/10.1001/jamaoncol.2018.5188 